\title{
$p$-process nucleosynthesis via proton-capture reactions in thermonuclear supernovae explosions
}

\author{
Anne Endres ${ }^{1, a}$, C. Arda ${ }^{1}$, P. Erbacher ${ }^{1}$, J. Glorius ${ }^{1,2}$, K. Göbel ${ }^{1}$, O. Hinrichs ${ }^{1}$, E. Mevius ${ }^{1}$, M. Reich ${ }^{1}$, K. Sonnabend ${ }^{1}$, \\ B. Thomas ${ }^{1}$, and T. Thomas ${ }^{1}$ \\ ${ }^{1}$ Institut für Angewandte Physik, Goethe Universität Frankfurt am Main, Germany \\ ${ }^{2}$ GSI Helmholtzzentrum für Schwerionenforschung $\mathrm{GmbH}$, Darmstadt, Germany
}

\begin{abstract}
Model calculations within the framework of the so-called $\gamma$ process show an underproduction of the $p$ nucleus with the highest isotopic abundace ${ }^{92} \mathrm{Mo}$. This discrepancy can be narrowed by taking into account the alternative production site of a type Ia supernova explosion. Here, the nucleus ${ }^{92}$ Mo can be produced by a sequence of proton-capture reactions. The amount of ${ }^{92}$ Mo nuclei produced via this reaction chain is most sensitive to the reactions ${ }^{90} \mathrm{Zr}(\mathrm{p}, \gamma)$ and ${ }^{91} \mathrm{Nb}(\mathrm{p}, \gamma)$. Both rates have to be investigated experimentally to study the impact of this nucleosynthesis aspect on the long-standing ${ }^{92}$ Mo-problem. We have already measured the proton-capture reaction on ${ }^{90} \mathrm{Zr}$ using high-resolution in-beam $\gamma$-ray spectroscopy. In this contribution, we will present our preliminary results of the total cross sections as well as the partial cross sections. Furthermore, we plan to measure the ${ }^{91} \mathrm{Nb}(\mathrm{p}, \gamma)$ reaction soon. Due to the radioactive target material, the ${ }^{91} \mathrm{Nb}$ nuclei have to be produced prior to the experiment. The current status of this production will be presented in this contribution.
\end{abstract}

\section{Introduction}

The elements heavier than iron are mainly created by neutron-capture reactions in the $s$ and $r$ processes [1, 2]. Nevertheless, between 30 and 35 isotopes, the so-called $p$ nuclei, are bypassed by the reaction paths of both processes [3]. Their existence can be explained by several processes summarized as $p$ process [4-8].

The relative abundances of most $p$ nuclei can be explained by the so-called $\gamma$ process [9]. Nevertheless, the $\gamma$ process cannot account for the high isotopic abundance of the neutron-magic $p$ nucleus ${ }^{92}$ Mo of $I_{\%}=14.84 \%$ [10]. Different network calculations predict a production of ${ }^{92} \mathrm{Mo}$ by more than one order of magnitude below the expected yield. Hence, an additional production mechanism is needed. A chain of radiative proton-capture reactions may contribute to the stable and long-lived neutron-magic $N=50$ nuclei during a supernova of type Ia $[11,12]$. The proton-capture cross sections on ${ }^{90} \mathrm{Zr}$ and ${ }^{91} \mathrm{Nb}$ determine the abundance of ${ }^{92} \mathrm{Mo}$ : their reaction rate is lower compared to the other $N=50$ isotopes in this chain, and all instable ${ }^{91} \mathrm{Nb}$ nuclei have to be produced during the supernova explosion itself. To investigate the underproduction of ${ }^{92} \mathrm{Mo}$ in model calculations of type Ia supernova explosions, reliable reaction rates of ${ }^{90} \mathrm{Zr}(\mathrm{p}, \gamma)$ and ${ }^{91} \mathrm{Nb}(\mathrm{p}, \gamma)$ are needed.

\section{Investigation of the ${ }^{90} \mathrm{Zr}(\mathbf{p}, \gamma){ }^{91} \mathrm{Nb}$ reaction}

There are three experimental data sets for the ${ }^{90} \mathrm{Zr}(\mathrm{p}, \gamma){ }^{91} \mathrm{Nb}$ reaction available in literature. Roughton et al. determined astrophysical reaction rates in a thick target yield measurement [13]. These values could be confirmed by a $4 \pi$ in-beam summing crystal measurement by A. Spyrou et al. [14]. Nevertheless, a measurement using $\gamma$-ray spectroscopy by C. E. Laird et al. disagrees with the two other data sets for the total cross section [15]. In order to solve this discrepancy, we measured this reaction again using high-resolution in-beam $\gamma$-ray spectroscopy. This method allows to investigate the total cross section as well as partial cross sections. To date, partial cross sections of ${ }^{90} \mathrm{Zr}(\mathrm{p}, \gamma)$ are not published at all.

The experiment was performed at the Institut für Kernphysik of the Universität zu Köln, Germany, where we used HORUS with a target chamber optimised for nuclear astrophysics experiments [16]. Thirteen high-purity germanium (HPGe) detectors were placed at five angles relative to the beam axis in order to determine the angular distribution of the prompt $\gamma$ rays of the excited reaction products. The angular distribution of the $\gamma$ transitions are mandatory to obtain the number of $\gamma$ rays emitted in the solid angle for each transition. Since ${ }^{91} \mathrm{Nb}$ has an isomer at $104.62 \mathrm{keV}$ [17], the sum of all ground state transitions (except the transition between isomer and groundstate) and of all transitions terminating in the isomer yield the total cross section. Furthermore, the depopulation of the so-called entry state can be investigated in order

a e-mail: endres@iap.uni-frankfurt.de 
to obtain partial cross sections. More details about this experimental method can be found in Ref. [18].

The ${ }^{90} \mathrm{Zr}(\mathrm{p}, \gamma)$ reaction was investigated for energies between $E_{\mathrm{p}}=3.6 \mathrm{MeV}$ and $5.1 \mathrm{MeV}$. A measurement as low in energy as $E_{\mathrm{p}}=2.5 \mathrm{MeV}$ is planned in near future. We used isotopically enriched material consisting of $97.65(10) \%{ }^{90} \mathrm{Zr}$. Besides other zirconium isotopes, $0.96(5) \%{ }^{91} \mathrm{Zr}$ is included. The two reactions ${ }^{90} \mathrm{Zr}(\mathrm{p}, \gamma)$ and ${ }^{91} \mathrm{Zr}(\mathrm{p}, \mathrm{n})$ both produce ${ }^{91} \mathrm{Nb}$ above a proton energy of about $2 \mathrm{MeV}$. The contribution of both reactions can be disentangled by performing measurements with another target with different enrichments $\left(5.99(10) \%\right.$ in ${ }^{90} \mathrm{Zr}$ and $89.20(10) \%$ in ${ }^{91} \mathrm{Zr}$ ) at the same energies. Details about this procedure can be found in the contribution of P. Erbacher in this volume.

Figure 1 shows our preliminary results for the total cross section in comparison with the cross section data by Refs. $[14,15]$. The total cross sections published in Laird et al. include estimated cross sections of the unobserved $\gamma$ rays terminating in the ground or first excited state. Our experimental results agree with the data of Laird et al. very well. The data by Spyrou et al. yields higher cross sections, especially at higher energies.

Furthermore, the experimental results are compared with theoretical cross section predictions by the TALYS code $[19,20]$. The cross section predictions are sensitive to the $\gamma$-ray strength function and the proton+nucleus optical model potential. Hence, the cross section predictions were calculated with all variations available in TALYS for this nuclear physics input. Our experimental results agree very well with the theoretical predictions using the default settings of the TALYS code. These calculations use the proton+nucleus optical model potential of Ref. [21] and the $\gamma$-ray strength function of Ref. [22].

Besides the total cross sections, we have analysed partial cross sections as well. Up to date, we have obtained partial cross sections for the transitions terminating in the ground state and three excited states. As an example, the partial cross section to the ground state is depicted in Fig. 2. It is compared to TALYS calculations using the default settings [21, 22].

\section{Production of ${ }^{91} \mathrm{Nb}$}

The ${ }^{91} \mathrm{Nb}(\mathrm{p}, \gamma)$ reaction might play an important role for the production of the $p$ nucleus ${ }^{92} \mathrm{Mo}$. It is possible to perform this experiment using standard kinematics due to the long half-life of the isotope ${ }^{91} \mathrm{Nb}\left(t_{1 / 2}=680 \mathrm{a}\right)$. In a first step, the target material has to be produced. An amount of $10^{16}$ nuclei should be sufficient to perform the experiment taking into account the proton-beam intensity and detection system under construction at FRANZ, Frankfurt a. M., Germany [29]. One way to produce ${ }^{91} \mathrm{Nb}$ is the bombardment of ${ }^{92} \mathrm{Mo}$ with protons. Three reactions produce ${ }^{91} \mathrm{Nb}$ either directly or via $\beta$-decay of the reaction products: ${ }^{92} \mathrm{Mo}(\mathrm{p}, 2 \mathrm{p}),{ }^{92} \mathrm{Mo}(\mathrm{p}, \mathrm{pn})$, and ${ }^{92} \mathrm{Mo}(\mathrm{p}, 2 \mathrm{n})$.

In order to determine the number of produced ${ }^{91} \mathrm{Nb}$ after irradiation, the induced radioactive decays have to be observed. ${ }^{91} \mathrm{Nb}$ has an isomer at an energy of $104.62 \mathrm{keV}$

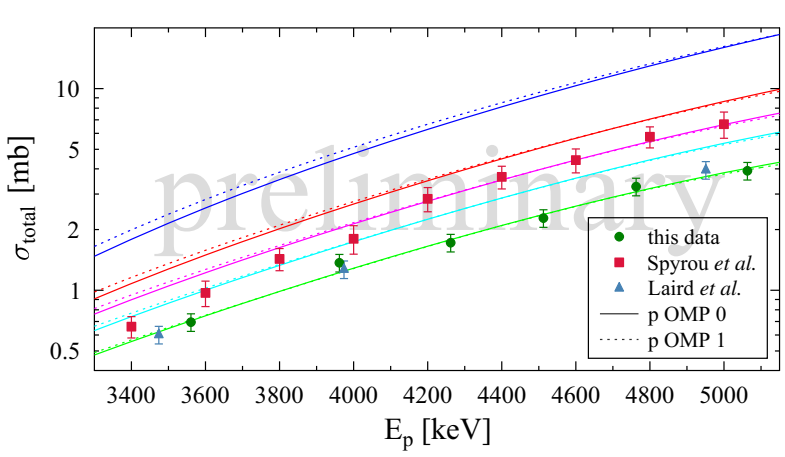

Figure 1. Experimental total cross section of the ${ }^{90} \mathrm{Zr}(\mathrm{p}, \gamma)$ reaction. Besides our data, three data sets are available in literature. The data of Laird et al. agrees very well with our values. However, the measurement by Syprou et al. yields larger cross sections, especially at higher proton energies. Furthermore, cross section predictions of the TALYS code are depicted. All combinations of proton+nucleus optical model potentials and $\gamma$-ray strength functions were used. Solid lines are calculated using the optical potential of Ref. [21], whereas calculations using the potential of Ref. [23] are depicted as dashed line. The different colours label the $\gamma$-ray strength function used for the calculations: lightgreen [22], blue [24, 25], cyan [26], magenta [27], and red [28].

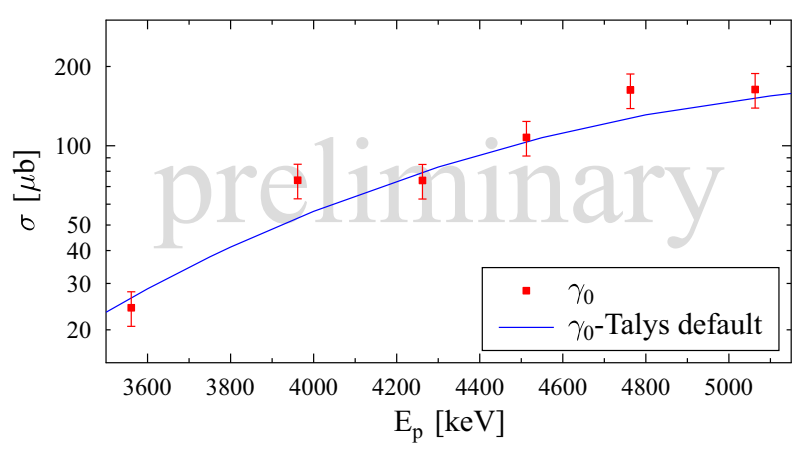

Figure 2. Experimental partial cross section ${ }^{90} \mathrm{Zr}\left(\mathrm{p}, \gamma_{0}\right)$ to the ground state of ${ }^{91} \mathrm{Nb}$. Furthermore, a cross section prediction of the TALYS code using the default settings is depicted.

with a half-life of $t_{1 / 2}=60.86 \mathrm{~d}$. Its decay can be investigated by detecting two $\gamma$ rays with energies of $104.62 \mathrm{keV}$ and $1204.67 \mathrm{keV}$ [17]. After the decay of the ground state, no $\gamma$ rays are emitted. Therefore, only X-rays can to be detected in order to determine the number of ${ }^{91} \mathrm{Nb}$ nuclei.

A thick target with a high enrichment in ${ }^{92} \mathrm{Mo}$ is needed for the production of $10^{16}{ }^{91} \mathrm{Nb}$ nuclei. The number of produced nuclei cannot be determined via $\gamma$-ray spectroscopy of the X-rays. Only a small fraction of the X-rays can escape from the thick target due to selfabsorption. Since no experimental data is available for the energy-dependent production cross sections of ${ }^{91} \mathrm{Nb}$, we decided to measure them prior to the target production runs. 
The measurement of the production cross sections was performed at the cyclotron of the Physikalisch-Technische Bundesanstalt at Braunschweig, Germany. Naturally composed targets were bombared with protons with energies of $E_{\mathrm{p}}=15,16,17,18$, and $19 \mathrm{MeV}$. Two activation runs were performed for each energy: a short activation of about 15 minutes and a long activation of about $8 \mathrm{~h}$. The duration of the short activation is in the order of the halflife of ${ }^{91} \mathrm{Mo}$ and is used to determine the ${ }^{92} \mathrm{Mo}(\mathrm{p}, \mathrm{pn}){ }^{91} \mathrm{Mo}$ cross section. The long activations were used to determine the total yield of ${ }^{91} \mathrm{Nb}$ via $\mathrm{x}$-ray detection for the ground state and $\gamma$-ray detection for the isomer.

The experiment was performed in July 2014, hence, the spectroscopy is still in progress at the Institut für Angewandte Physik at Frankfurt a. M., Germany. Estimates based on the first spectra show that a sufficient production of ${ }^{91} \mathrm{Nb}$ via proton-induced reactions on ${ }^{92} \mathrm{Mo}$ is possible [30].

\section{Acknowledgments}

We thank the group of A. Zilges and the accelerator staff at Universität zu Köln for their enormous support during the ${ }^{90} \mathrm{Zr}(\mathrm{p}, \gamma)$ beam time. Furthermore, we thank U. Giesen and the accelerator staff at Physikalisch-Technische Bundesanstalt at Braunschweig for the collaboration during the bombardment of ${ }^{\text {nat }}$ Mo. This work is supported by Deutsche Forschungsgemeinschaft (SO907/2-1) and by the HIC for FAIR within the framework of LOEWE launched by the State of Hesse, Germany. J. G. acknowledges support by Nautilus, K. G. by HGS-HIRE.

\section{References}

[1] F. Käppeler, R. Gallino, S. Bisterzo, W. Aoki, Rev. Mod. Phys. 83, 157 (2011)

[2] M. Arnould, S. Goriely, K. Takahashi, Phys. Rep. 450, 97 (2007)

[3] D. L. Lambert, Astron. Astroph. Rev. 3, 201 (1992)

[4] S. E. Woosley, W. M. Howard, Astrophys. J. Suppl. 36, 285 (1978)

[5] H. Schatz, A. Aprahamian, J. Görres, M. Wiescher, T. Rauscher, J. Rembges, F.-K. Thielemann, B. Pfeiffer, P. Möller, K.-L. Kratz, H. Herndl, B. Brown, H. Rebel, Phys. Pep. 294, 167 (1998)

[6] S. Goriely, J. José, M. Hernanz, M. Rayet, M. Arnould, Astron. Astroph. 383, L27 (2002)

[7] C. Fröhlich, G. Martinez-Pinedo, M. Liebendörfer, F.K. Thielemann, E. Bravo, W. Hix, K. Langanke, N. Zinner, Phys. Rev. Lett. 96, 142502 (2006)

[8] S. Woosley, D. Hartmann, R. Hoffman, W. Haxton, Astrophs. J. 356, 272 (1990)

[9] M. Arnould, S. Goriely, Phys. Rep. 384, 1 (2003)
[10] P. De Bievre and P. D. P. Taylor, Int. J. Mass Spectrom. Ion Phys. 123, 149 (1993)

[11] M. Kusakabe, N. Iwamoto, K. Nomoto, Astrophys. J. 726, 25 (2011)

[12] C. Travaglio, F. Röpke, R. Gallino, W. Hillebrandt, Astrophys. J. 739, 93 (2011)

[13] N. A. Roughton, M. R. Fritts, R. J. Peterson, C. S. Zaidins, C. J. Hansen, At. Data Nucl. Data Tables 23, 177 (1979)

[14] A. Spyrou, S. J. Quinn, A. Simon, T. Rauscher, A. Battaglia, A. Best, B. Bucher, M. Couder, P. A. DeYoung, A. C. Dombos, X. Fang, J. Görres, A. Kontos, Q. Li, L. Y. Lin, A. Long, S. Lyons, B. S. Meyer, A. Roberts, D. Robertson, K. Smith, M. K. Smith, E. Stech, B. Stefanek, W. P. Tan, X. D. Tang, M. Wiescher, Phys. Rev. C 88, 045802 (2013)

[15] C. E. Laird, D. Flynn, R. L. Hershberger, F. Gabbard, Phys. Rev. C 35, 1265 (1987)

[16] L. Netterdon, V. Derya, J. Endres, C. Fransen, A. Hennig, J. Mayer, C. Müller-Gatermann, A. Sauerwein, P. Scholz, M. Spieker, A. Zilges, Nucl. Instr. and Meth. Phys. Res. A 754, 94 (2014)

[17] C. M. Baglin, Nuclear Data Sheets 114 (2013) 1293, online version (http://www.nndc.bnl.gov, $24^{\text {th }}$ September 2014)

[18] A. Sauerwein, J. Endres, L. Netterdon, A. Zilges, V. Foteinou, G. Provatas, T. Konstaninopoulos, M. Axiotis, S. F. Ashleys, S. Harissopulos, T. Rauscher, Phys. Rev. C 86, 035802 (2012)

[19] A. J. Koning, S. Hilaire, M. C. Duijvestjin, Proceedings of the International Conference on Nuclear Data for Science and Technology-ND2004 (AIP Conference Proceedings 769, Santa Fe, 2004) 1154

[20] TALYS-1.4, User Manual, http://www.talys.eu

[21] A. J. Koning and J. P. Delaroche, Nucl. Phys. A713, 231 (2003)

[22] J. Kopecky and M. Uhl, Phys. Rev. C 41, 1941 (1990)

[23] E. Bauge, J.P. Delaroche, and M. Girod, Phys. Rev. C 63, 024607 (2001)

[24] D. M. Brink, Nucl. Phys. 4, 215 (1957)

[25] P. Axel, Phys. Rev. C 126, 671 (1962)

[26] S. Goriely and E. Khan, Nucl. Phys. A706, 217 (2002)

[27] S. Goriely, E. Khan, and M. Samyn, Nucl. Phys. A739, 331 (2004)

[28] S. Goriely, Phys. Lett. B 436, 10 (1998)

[29] R. Reifarth, C. Lederer, and F. Käppeler, J. Phys. G 41, 053101 (2014)

[30] B. Thomas, K. Sonnabend, C. Arda, R. Dressler, A. Endres, P. Erbacher, S. Fiebiger, U. Giesen, J. Glorius, O. Hinrichs, H.-K. Kim, M. Reich, S. Schmidt, and D. Schumann, PoS(NIC XIII), 118 (2014) 
